accelerated titration design was utilized for the initial dose escalation followed by a modified $3+3$ design and the option to back-fill cohorts. Dose-limiting toxicities (DLTs) were reported during the first cycle of each schedule. The primary study objectives include the safety profile and RP2D of PRS343. Secondary objectives include ORR and DCR, PD biomarker response and PK profile. PD response was assessed in tumor biopsies (CD8 $+\mathrm{T}$ cell IHC) pre- and post- PRS-343 treatment.

Results 51 patients (median age 61.2 years, 61\% female, 82\% caucasian, $57 \%$ with more than three lines of prior therapy) with a variety of solid tumor indications [gastric/GEJ $(n=19)$; BC $(n=12)$; gynecological cancer $(n=6)$; CRC $(n=5)$; BTC $(\mathrm{n}=4)$; UC $(\mathrm{n}=2)$; melanoma, pancreatic and salivary duct $(n=1$ each)] have been treated with PRS-343. Based on pharmacokinetic analyses and observed kinetics of the CD8 $+\mathrm{T}$ cell expansion post-treatment, the low end of the active dose range is considered $2.5 \mathrm{mg} / \mathrm{kg}$. 19 patients treated at active dose levels before the data cut-off on 09-06-2019 were evaluable for response [DCR 58\% (11\% confirmed PR) as per RECIST 1.1]. At the active doses, we observed significant and pronounced post-treatment expansion of CD8 $+\mathrm{T}$ cells particularly in the tumor nests, consistent with the MoA of PRS343, while there was no increase in the doses below $2.5 \mathrm{mg} /$ $\mathrm{kg}$. The post-treatment expansion of CD8 $+\mathrm{T}$ cells was more pronounced in patients with a confirmed PR or prolonged SD. PRS-343 was very well tolerated, with no SAEs reported. The most frequent TRAEs were fatigue (9\%), chills $(6 \%)$ and diarrhea $(5 \%)$ of mild to moderate severity. None qualified as a DLT.

Conclusions PRS-343 is the first molecule of its kind to demonstrate encouraging evidence of safety and clinical benefit with a correlative PD effect in a heavily pre-treated population. These initial data suggest that PRS-343, the first 4-1BB bispecific to enter clinical development, merits further investigation in clinical trials.

Trial Registration NCT03330561

\section{PHASE 1 STUDY OF AN ANTI-CD27 AGONIST AS MONOTHERAPY AND IN COMBINATION WITH PEMBROLIZUMAB IN PATIENTS WITH ADVANCED SOLID TUMORS} ${ }^{3}$ Elliot Chartash, ${ }^{3}$ Fang Liu, ${ }^{3}$ Claire Li, ${ }^{3}$ Richard Wnek, ${ }^{4}$ Manish Patel. ${ }^{1}$ Oncology Institute, Sheba Medical Center, Ramat-Gan, Israel, Ramat-Gan, Israel; ${ }^{2}$ Antoni van Leeuwenhoek Hospital, Amsterdam, Netherlands, Amsterdam, Netherlands; ${ }^{3}$ Merck and Co., Inc., Kenilworth, NJ, USA, Kenilworth, NJ, USA; ${ }^{4}$ Florida Cancer Specialists/Sarah Cannon Research Institute, Sarasota, FL, USA

\subsection{6/LBA2019.3}

Background MK-5890 is a humanized agonist monoclonal antibody that binds to CD27 to provide a costimulatory signal that enhances T-cell-mediated responses. This first-in-human phase 1 study of MK-5890 evaluated the safety and efficacy of escalating doses of MK-5890 as monotherapy and in combination with pembrolizumab in patients with advanced solid tumors.

Methods Key eligibility criteria included histologically or cytologically confirmed advanced solid tumor, measurable disease by RECIST v1.1, and ECOG PS $\leq 1$. MK-5890 was tested alone (dose range, 2-700 $\mathrm{mg}$ ) or with pembrolizumab (fixed dose, $200 \mathrm{mg}$ ). Patients with disease progression following MK-5890 monotherapy were eligible to cross over to combination treatment. The primary objective was safety and tolerability. Objective response rate by investigator per RECIST v1.1 was also evaluated. The database cutoff for this analysis was May 30, 2019.

Results Of 44 patients enrolled, 25 received MK-5890 and 19 received MK-5890 plus pembrolizumab; their median age was 59.0 years, $61.4 \%$ were female, $47.7 \%$ had ECOG PS 1 , and $13.6 \%$ previously received immune checkpoint inhibitor therapy. In the initial phase, dose-limiting toxicities (DLTs) were reported in 3 patients receiving MK-5890 and 1 patient receiving MK-5890 plus pembrolizumab; all DLTs were associated with infusion-related adverse events. Maximum tolerated dose was defined. Treatment-related adverse events (TRAEs) were reported in 40 patients (90.9\%): 22 patients $(88.0 \%)$ receiving MK-5890 and 18 patients (94.7\%) receiving MK5890 plus pembrolizumab. The most common TRAEs were fatigue $(28.0 \%)$ and infusion-related reactions $(28.0 \%)$ with MK-5890 and fatigue (36.8\%) and pruritus (31.6\%) with MK5809 plus pembrolizumab. Grade 3-4 TRAEs were reported in 10 patients $(22.7 \%)$ : 6 patients $(24.0 \%)$ receiving MK-5890 and 4 patients (21.1\%) receiving MK-5890 plus pembrolizumab; no grade 5 events were observed. One patient (4.0\%) achieved a partial response (PR) with MK-5890 and 1 patient (5.3\%) achieved a PR with MK-5890 plus pembrolizumab. Fourteen patients entered the crossover phase to receive MK5890 plus pembrolizumab. In the crossover phase, no DLTs were reported. TRAEs were reported in 12 patients $(85.7 \%)$; the most common were pruritus $(21.4 \%)$, rash $(21.4 \%)$, and headache $(14.3 \%)$. One patient $(7.1 \%)$ reported grade $3-4$ TRAEs of increased amylase and increased lipase; no grade 5 events were observed. Two patients (14.3\%) achieved a complete response and 2 patients (14.3\%) achieved a PR.

Conclusions Treatment with MK-5890, alone and in combination with pembrolizumab, demonstrated an acceptable safety profile. Early antitumor activity was observed in patients with advanced solid tumors in both monotherapy and combination therapy arms.

\section{DURABLE RESPONSES IN ANTI-PD-1 REFRACTORY MELANOMA FOLLOWING INTRATUMORAL INJECTION OF A TOLL-LIKE RECEPTOR 9 (TLR9) AGONIST, CMP-001, IN COMBINATION WITH PEMBROLIZUMAB}

${ }^{1}$ Mohammed Milhem, ${ }^{1}$ Yousef Zakharia, ${ }^{2}$ Diwakar Davar, ${ }^{3}$ Elizabeth Buchbinder, ${ }^{4}$ Theresa Medina, ${ }^{5}$ Adil Daud, ${ }^{6}$ Antoni Ribas, ${ }^{7}$ Jiaxin Niu, ${ }^{8}$ Geoffrey Gibney, ${ }^{9}$ Kim Margolin, ${ }^{10}$ Anthony Olszanski, ${ }^{9}$ Interjit Mehmi, ${ }^{11}$ Takami Sato, ${ }^{12}$ Montaser Shaheen, ${ }^{13}$ Aaron Morris, ${ }^{13}$ David Mauro, ${ }^{6}$ Katie Campbell, ${ }^{2}$ Riyue Bao, ${ }^{1}$ George Weiner, ${ }^{1}$ Jason Luke, ${ }^{13}$ Arthur Krieg, ${ }^{2}$ John Kirkwood*. 'University of lowa, lowa City, IA, USA; ${ }^{2}$ University of Pittsburgh Medical Center, Pittsburgh, PA, USA; ${ }^{3}$ Dana Farber Cancer Institute, Boston, MA, USA; ${ }^{4}$ University of Colorado Denver, Aurora, CO, USA; ${ }^{5}$ University of California San Francisco, San Francisco, CA, USA; ${ }^{6}$ University of California Los Angeles, Los Angeles, CA, USA; ${ }^{7}$ Banner $M D$ Anderson Cancer Center, Gilbert, $A Z$, USA; ${ }^{8}$ Georgetown University, Washington, DC, USA; ${ }^{9}$ City of Hope, Duarte, CA, USA; ${ }^{10}$ Fox Chase Cancer Center, Phildelphia, PA, USA; ${ }^{11}$ Thomas Jefferson University, Philadelphia, PA, USA; ${ }^{12}$ University of Arizona, Tucson, $A Z$, USA; ${ }^{13}$ Checkmate Pharmaceuticals, Cambridge, MA, USA

\subsection{6/LBA2019.4}

Background Intratumoral (IT) injection of CMP-001, a CpG-A TLR9 agonist packaged within a virus-like particle, is designed 
Abstract 085 Table 1 Advanced anti-PD-1 Refractory melanoma patient population by treatment allocation

\begin{tabular}{|c|c|c|c|c|c|c|c|c|}
\hline & \multicolumn{4}{|c|}{$\begin{array}{c}\text { CMP-001+ Pembrolizumab } \\
\mathrm{N}=144\end{array}$} & \multirow{2}{*}{\multicolumn{4}{|c|}{$\begin{array}{l}\text { CMP-001 monotherapy } \\
\qquad \mathrm{N}=23\end{array}$}} \\
\hline & \multirow{2}{*}{$\begin{array}{c}\text { Dose } \\
\text { escalation } \\
\mathrm{N}=44\end{array}$} & \multicolumn{2}{|c|}{$\begin{array}{l}\text { First expansion } \\
\quad \mathrm{N}=69\end{array}$} & \multirow{2}{*}{$\begin{array}{c}\text { Second } \\
\text { expansion } \\
\mathrm{N}=31\end{array}$} & & & & \\
\hline & & $\mathrm{N}=8^{*}$ & $\mathrm{~N}=61$ & & $\mathrm{~N}=13$ & $\mathrm{~N}=1$ & $\mathrm{~N}=4$ & $\mathrm{~N}=5$ \\
\hline $\begin{array}{l}\text { CMP-001 } \\
\text { Treatment: }\end{array}$ & CMP-001^ & $\begin{array}{l}\text { CMP-001 } \\
\text { initially* }\end{array}$ & $\begin{array}{l}\text { Diluted } \\
\text { CMP- } \\
001^{*}\end{array}$ & $\begin{array}{l}\text { CMP-001 } \\
(10 \mathrm{mg})\end{array}$ & $\begin{array}{l}\text { CMP-001 } \\
(10 \mathrm{mg})\end{array}$ & $\begin{array}{l}\text { CMP-001 } \\
\text { initially } \\
(10 \mathrm{mg}) \S\end{array}$ & \begin{tabular}{|l|} 
Diluted \\
CMP-001 \\
(5 mg) \\
\end{tabular} & $\begin{array}{l}\text { Diluted CMP- } \\
001 \text { initially } \\
(5 \mathrm{mg})\end{array}$ \\
\hline $\begin{array}{c}\text { ORR } \\
(95 \% \mathrm{CI}):\end{array}$ & $\begin{array}{c}22.7 \% \\
(11.5 \%- \\
37.8 \%)\end{array}$ & $\begin{array}{l}37.5 \% \\
(8.5 \%- \\
75.5 \%)\end{array}$ & $\begin{array}{l}11.5 \% \\
(4.8 \%- \\
22.3 \%)\end{array}$ & $\begin{array}{c}25.8 \% \\
(11.9 \%- \\
44.6 \%)\end{array}$ & $15.4 \%$ & $0 \%$ & $0 \%$ & $60 \%$ \\
\hline
\end{tabular}

"The first 8 patients in the combination therapy arm of the original ("First") expansion cohort were initiated on CMP-001 therapy using the standard drug product buffer at $10 \mathrm{mg}$. During their treatment course, on-site dilution of CMP-001 from the stock concentration of $6 \mathrm{mg} / \mathrm{mL}$ to a final concentration of $1 \mathrm{mg} / \mathrm{mL}$ ( $5 \mathrm{mg}$ dose) was initiated using normal saline, and 6 of these 8 patients were switched to that formulation at various times during their treatment. The remaining 61 patients in the first expansion cohort were initiated on therapy using the on-site dilution of CMP-001 at $5 \mathrm{mg}$.

$\wedge$ dose levels: $1 \mathrm{mg}(\mathrm{N}=3), 3 \mathrm{mg}(\mathrm{N}=16), 5 \mathrm{mg}(\mathrm{N}=9), 7.5 \mathrm{mg}(\mathrm{N}=6), 10 \mathrm{mg}(\mathrm{N}=10)$

$\S 1$ patient in the monotherapy arm was initiated on treatment with CMP-001, but then switched to on-site dilution in saline at week 10

\5 patients in the monotherapy arm were initiated on treatment with saline-diluted CMP-001, but then switched to undiluted CMP-001 at time points ranging from weeks 4 to 13

The purpose of this on-site dilution step was to increase the volume of injection, improving the distribution of the drug in the tumor. Initial studies showed no adverse effects of on-site dilution on CMP-001 efficacy. However, subsequent in vivo preclinical studies revealed that the on-site dilution step significantly reduces the anti-tumor efficacy of CMP-001, and so a second expansion cohort was enrolled, using the original undiluted buffer composition of CMP-001

to activate tumor-associated plasmacytoid dendritic cells, inducing an interferon-rich tumor microenvironment and anti-tumor $\mathrm{CD} 8+\mathrm{T}$ cell responses.

Methods CMP-001-001 is an ongoing Phase $1 \mathrm{~b}$ trial evaluating the safety and efficacy of CMP-001 in combination with pembrolizumab (Part 1; $\mathrm{N}=144$ ) or alone (Part 2; $\mathrm{N}=$ 23) in patients with advanced melanoma resistant to prior anti-PD-1 therapy (Tables 1). CMP-001 is administered IT into accessible lesion(s) either with, or without on-site saline dilution (table 1), and response assessed by RECIST v1.1. Monotherapy patients who progress can be rolled over onto combination therapy and continue on study. Baseline and ontherapy serum is analyzed for cytokines, and immunohistochemistry and RNA-Seq are performed on available tumor biopsies.

Results Adverse events (AEs) attributed to CMP-001 in combination with pembrolizumab or as monotherapy consisted predominately of transient low-Grade flu-like symptoms and injection site reactions: Grade 3+ related AEs were reported in $33 \%$ of patients treated with combination therapy and $22 \%$ of patients with monotherapy.

The Objective Response Rate (ORR) with undiluted CMP001 in combination with pembrolizumab was $24 \%(18 / 75$; 95\% confidence interval: 15\%-35\% (table 1); on-site dilution of CMP-001 was associated with a substantial decrease in ORR to $12 \%(7 / 61 ; 95 \%$ confidence interval: $5 \%-22 \%$ (table 1). Three additional patients had a delayed partial response after an initial period of disease progression. Anti-tumor response was comparable between injected and uninjected lesions. The median duration of response to combination therapy has not been reached. The ORR to CMP-001 monotherapy was $22 \%(5 / 23 ; 95 \%$ confidence interval: $7 \%-44 \%$ (table $1)$; time from last anti-PD-1 therapy before CMP-001 was 1.5 to $>20$ months in responders; 3 of the patients responding to
CMP-001 monotherapy achieved PR at the first evaluation, but progressed by the second evaluation.

Serum and tumor biopsy translational studies in the patients receiving combination therapy supported the proposed mechanism of TLR9 activation and identified a possible association between induction of serum CXCL10 and response.

Conclusions IT CMP-001 alone and in combination with pembrolizumab appears well tolerated, can reverse resistance to anti-PD-1 therapy, and can produce deep and durable clinical responses in patients with advanced melanoma.

Ethics Approval CMP-001-001 was centrally approved by the WCG-WIRB, WIRB approval tracking number 20152597.

\section{Poster Presentations}

\section{Biomarkers, immune monitoring, and novel technologies}

\section{P851 IDENTIFYING POTENTIAL PREDICTIVE BIOMARKERS FROM PLASMA EXOSOMES AND ADOPTIVE T CELLS THAT DIFFERENTIATE SHORT AND LONG-TERM METASTATIC NASOPHARYNGEAL CANCER SURVIVORS TREATED WITH CHEMOTHERAPY AND VIRUS-SPECIFIC T CELLS}

${ }^{1}$ Timothy Shuen, ${ }^{1}$ WANG Who-Whong, ${ }^{2}$ Han Chong Toh*, 'Janice Lim, ${ }^{1}$ Cherlyn Tan, ${ }^{1}$ Jennie Kosasih, ${ }^{1}$ Rachael Cheong. ${ }^{1}$ National Cancer Centre Singapore, Singapore; ${ }^{2}$ Tessa Therapeutics Ltd, Singapore, Singapore

\subsection{6/LBA2019.5}

